This paper appeared in Proceedings of the SPIE Conference on Independent Component Analyses, Wavelets, Neural Networks, Biosystems, and Nanoengineering IX, Orlando, FL., April 2011.

\title{
Three-Dimensional Shearlet Edge Analysis
}

\author{
David A. Schug ${ }^{a}$, Glenn R. Easley ${ }^{b}$, Dianne P. O'Leary ${ }^{a}$ \\ ${ }^{a}$ University of Maryland, College Park, Maryland \\ ${ }^{b}$ System Planning Corporation, Arlington, Virginia
}

\begin{abstract}
Volumetric data acquisition and increasingly massive data storage have increased the need to develop better analysis tools for three-dimensional data sets. These volumetric data sets can provide information beyond that contained in standard two-dimensional images. Common strategies to deal with such data sets have been based on sequential use of two-dimensional analysis tools. In this work, we propose using an extension of the wavelet transform known as the shearlet transform for the purpose of edge analysis and detection in three-dimensions. This method takes advantage of the shearlet transform's improved capability to identify edges compared to wavelet-based approaches.
\end{abstract}

Keywords: shearlets, wavelets

\section{INTRODUCTION}

The desire to locate perceptible changes in image intensity or edges has received a considerable amount of attention corresponding with the ongoing need to detect and estimate features. For a given image $I:=[0,1]^{2} \rightarrow$ $[0,1]$, the collection of edge intensities are

$$
E=\left\{e \in[0,1]^{2}:|\nabla I(e)| \geq h\right\},
$$

where the magnitude of the gradient of $I$ is above a scalar threshold $h \in[0,1]$ for each element $e$. Edge detection applications include imagery collected from medical sensors and surveillance video, and many others. Automating the extraction of image features and detecting small but important jumps in intensity has proven difficult with many problems yet to be resolved. Real world data often provides many illumination changes, occlusions, complicated clutter, and noise that challenges the robustness of the latest technology. Even under ideal conditions, edge features are blurred or lost for edges that run close together. To mitigate the effects of noise, the image should first be smoothed with a low-pass averaging filter or a Gaussian filter to remove higher frequency information. This is accomplished by choosing a particular standard deviation $\sigma$ for a Gaussian filter $G_{\sigma}$ and convolving it with the image $I$ to generate a smoothed image

$$
I_{\sigma}=I * G_{\sigma} .
$$

The drawback to this step is choosing the correct image specific standard deviation. There is a delicate balance between removing unnecessary noise and possibly losing information. Multi-dimensional data sets add an additional degree of freedom for edges to change directions. Two-dimensional wavelet and shearlet approaches have proven effective in isolating edges at different scales. The continuous wavelet transform of image $I$ is given by

$$
W_{\varphi} I(M, \tau)=\left\langle I, \varphi_{M, \tau}\right\rangle=a^{-1} \int_{\mathbb{R}} I(x) \overline{\varphi\left(a^{-1}(x-\tau)\right)} d x,
$$

where $M=a \mathbb{I}$ is an the identity matrix $\mathbb{I}$ scaled by $a>0$. The analysis functions

$$
\varphi_{M, \tau}(e)=|\operatorname{det} M|^{-\frac{1}{2}} \varphi\left(M^{-1}(e-\tau)\right), \quad \tau \in \mathbb{R}^{2},
$$

D.A.S: E-mail: dschug@umd.edu, G.R.E: E-mail: geasley@sysplan.com, D.P.O: E-mail: oleary@cs.umd.edu 
are well localized waveforms that can decompose images $I \in L^{2}\left(\mathbb{R}^{2}\right)$ so that

$$
I=\int_{\mathbb{R}^{2}}\left\langle I, \varphi_{M, \tau}\right\rangle \varphi_{M, \tau} d \tau
$$

By analyzing the the magnitudes of $\left\langle I, \varphi_{M, \tau}\right\rangle$ as a function of scale $a$, edge detection techniques can be developed. Unfortunately, the wavelet approach does not isolate any directional information. The wavelet transform is isotropic since the dilation factor is the same in all coordinate directions. Therefore, the wavelet has poor angular accuracy for edges that cross or have sharp curvature. Multi-directional shearlet analysis, on the other hand, has demonstrated better success at isolating edges whose orientations change in complicated ways. Given the analyzing function

$$
\varphi_{a, s, \tau}(e)=|\operatorname{det} M|^{-\frac{1}{2}} \varphi\left(M_{a, s}^{-1}(e-\tau)\right)
$$

where $M_{a s}=\left(\begin{array}{cc}a & -\sqrt{a} s \\ 0 & \sqrt{a}\end{array}\right), a \in \mathbb{R}^{+}, s \in \mathbb{R}$, and $\tau \in \mathbb{R}^{2}$, the continuous shearlet transform is defined as

$$
\mathcal{S H}_{\varphi}: I \rightarrow \mathcal{S H}_{\varphi} I(a, s, \tau)=\left\langle I, \varphi_{a, s, \tau}\right\rangle
$$

The matrix $M_{a s}$ performs both the shearing and anisotropic dilation. Likewise, these directional waveforms decompose images in $L^{2}\left(\mathbb{R}^{2}\right)$ so that

$$
I=\int_{\mathbb{R}^{2}} \int_{-\infty}^{\infty} \int_{0}^{\infty}\left\langle I, \varphi_{M_{a s}, \tau}\right\rangle \varphi_{M_{a s}, \tau} \frac{d a}{a^{3}} d s d \tau .
$$

These analyzing functions can represent scale, location and orientation of important image features such as edges.

The edges can be precisely characterized from the asymptotic decay of $\mathcal{S} \mathcal{H}_{\varphi} I(a, s, t)$. Given the collection of edge locations $E$, these characteristics are:

- If $\tau \notin E$, then $\mathcal{S H}_{\varphi} I(a, s, \tau)$ decays rapidly, as $a \rightarrow 0$, for each $s \in \mathbb{R}$. By rapid decay, we mean for any $N \in \mathbb{N}$ there is a $C_{N}>0$ such that $\left|\mathcal{S H}_{\varphi} I(a, s, \tau)\right| \leq C_{N} a^{N}$ as $a \rightarrow 0$.

- If $\tau \in E$ and $E$ is smooth near $\tau$, then $\left|\mathcal{S H}_{\varphi} I(a, s, \tau)\right|$ decays rapidly as $a \rightarrow 0$, for each $s \in \mathbb{R}$ unless $s=s_{0}$ is the normal orientation to $E$ at $\tau$ where $\mathcal{S H}_{\varphi} I(a, s, \tau) \sim a^{\frac{3}{4}}$, as $a \rightarrow 0$.

- If $\tau$ is a corner point of $E$ and $s=s_{0}$, and $s=s_{1}$ are normal orientations to the $E$ at $\tau$, then $\left|\mathcal{S H} \mathcal{H}_{\varphi} I\left(a, s_{0}, \tau\right)\right|$, $\left|\mathcal{S H}_{\varphi} I\left(a, s_{1}, \tau\right)\right| \sim a^{\frac{3}{4}}$ as $a \rightarrow 0$. For all other orientations the asymptotic decay of $\left|\mathcal{S H}_{\varphi} I(a, s, \tau)\right|$ is faster.

Up to this point, attention has been focused on one image at a time without regard for the possibility of processing an image sequence as a whole. Extending the wavelet edge detection routine to 3D data gives extra information that will likely help mitigate noise and improve identification. Our intent is to demonstrate the advantage of processing a cube of data representing video instead of sequentially on a frame by frame basis. The multi-scale, multi-directional aspect of the 3D shearlet transform should track edge information better than the 3D wavelet transform because of its added directional selectivity. It is interesting to note that, to the best of our knowledge, a 3D continuous wavelet transform has never been developed for this purpose before.

To understand the directional selectivity, one should realize that the 3D shearlet transform divides the spatial frequency domain into a number of filters shaped like hyper-trapezoids, as shown in Figure 1. Specifically, 3D shearlets are constructed by first restricting the subspace of $L^{2}\left(\mathbb{R}^{3}\right)$ to be $L^{2}\left(C^{(1)}\right)^{\vee}=\left\{f \in L^{2}\left(\mathbb{R}^{3}\right): \operatorname{supp} \widehat{f} \subset\right.$ $\left.C^{(1)}\right\}$, where $C^{(1)}$ is the horizontal cone in the frequency plane:

$$
C^{(1)}=\left\{\left(\eta_{1}, \eta_{2}, \eta_{2}\right) \in \mathbb{R}^{3}:\left|\eta_{1}\right| \geq 2,\left|\frac{\eta_{2}}{\eta_{1}}\right| \leq 1 \text { and }\left|\frac{\eta_{3}}{\eta_{1}}\right| \leq 1\right\} .
$$

We consider the shearlet group

$$
\Lambda^{(1)}=\left\{\left(M_{a s_{1} s_{2}, x}\right): 0 \leq a \leq \frac{1}{4},-\frac{3}{2} \leq s_{1} \leq \frac{3}{2},-\frac{3}{2} \leq s_{2} \leq \frac{3}{2}, x \in \mathbb{R}^{2}\right\}
$$


where $M_{a s_{1} s_{2}}=\left(\begin{array}{ccc}a-a^{1 / 2} s_{1}-a^{-1 / 2} s_{2} \\ 0 & a^{1 / 2} & 0 \\ 0 & 0 & a^{1 / 2}\end{array}\right)$. Then the following (see Proposition 2.1 from $^{3}$ ) defines the conditions on the function $\varphi^{(1)}$ to generate a continuous shearlet transform on $L^{2}\left(C^{(1)}\right)^{\vee}$, where the shearlet analyzing function is $\varphi_{a s_{1} s_{2} x}^{(1)}(y)=\left|\operatorname{det} M_{a s_{1} s_{2}}\right|^{-\frac{1}{2}} \varphi^{(1)}\left(M_{a s_{1} s_{2}}^{-1}(y-x)\right)$. For $\eta=\left(\eta_{1}, \eta_{2}, \eta_{3}\right) \in \mathbb{R}^{3}, \eta_{1} \neq 0$, let the function $\varphi^{(1)}$ be such that

$$
\hat{\varphi}^{(1)}(\eta)=\hat{\varphi}^{(1)}\left(\eta_{1}, \eta_{2}, \eta_{3}\right)=\hat{\varphi}_{1}\left(\eta_{1}\right) \hat{\varphi}_{2}\left(\frac{\eta_{2}}{\eta_{1}}\right) \hat{\varphi}_{2}\left(\frac{\eta_{3}}{\eta_{1}}\right) .
$$

If $\varphi_{1} \in L^{2}(\mathbb{R})$ satisfies the Calderòn condition

$$
\int_{0}^{\infty}\left|\hat{\varphi}_{1}(a \eta)\right|^{2} \frac{d a}{a}=1 \quad \text { for a.e. } \quad \eta \in \mathbb{R}
$$

with supp $\hat{\varphi}_{1} \subset\left[-2,-\frac{1}{2}\right] \cup\left[\frac{1}{2}, 2\right]$ and $\left\|\varphi_{2}\right\|_{L^{2}}=1$ with supp $\hat{\varphi}_{2} \subset\left[-\frac{\sqrt{2}}{4}, \frac{\sqrt{2}}{4}\right]$, then

$$
I(u)=\int_{\mathbb{R}^{3}} \int_{-\frac{3}{2}}^{\frac{3}{2}} \int_{-\frac{3}{2}}^{\frac{3}{2}} \int_{0}^{\frac{1}{4}}\left\langle I, \varphi_{a s_{1} s_{2}}^{(1)}\right\rangle \varphi_{a s_{1} s_{2}}^{(1)}(u) \frac{d a}{a^{4}} d s_{1} d s_{2} d u
$$

for all $I \in L^{2}\left(C^{(1)}\right)$.

In the frequency domain a shearlet $\varphi_{a s_{1} s_{2}}^{(1)}$ is defined by

$$
\hat{\varphi}_{a s_{1} s_{2} x}^{(1)}\left(\eta_{1}, \eta_{2}, \eta_{3}\right)=a \hat{\varphi}_{1}\left(a \eta_{1}\right) \hat{\varphi}_{2}\left(a^{-\frac{1}{2}}\left(\frac{\eta_{2}}{\eta_{1}}-s_{1}\right)\right) \hat{\varphi}_{2}\left(a^{-\frac{1}{2}}\left(\frac{\eta_{3}}{\eta_{1}}-s_{2}\right)\right) e^{-2 \pi i \eta x}
$$

Therefore, the functions $\hat{\varphi}_{a s_{1} s_{2} x}^{(1)}$ have supports in the sets:

$$
\left\{\left(\eta_{1}, \eta_{2}, \eta_{3}\right)\left|\eta_{1} \in\left[-\frac{2}{a},-\frac{1}{2 a}\right] \cup\left[\frac{1}{2 a}, \frac{2}{a}\right],\right| \frac{\eta_{2}}{\eta_{1}}-s_{1}\left|\leq \frac{\sqrt{2}}{4} a^{\frac{1}{2}},\right| \frac{\eta_{3}}{\eta_{1}}-s_{2} \mid \leq \frac{\sqrt{2}}{4} a^{\frac{1}{2}}\right\} .
$$

The frequency support is a pair of hyper-trapezoids that are symmetric with respect to the origin with orientation determined by slope parameters $s_{1}$ and $s_{2}$ and become elongated as $a \rightarrow 0$, as indicated in Figure 1 . This construction is further extended to cover the entire space $L^{2}\left(\mathbb{R}^{3}\right)$ by forming similar components valid on complementary cone regions for the generating functions $\varphi^{(2)}$ and $\varphi^{(3)}$ (see ${ }^{3}$ for more details). The superscript is then dropped and the notation $\varphi$ is simply used to denote the combined generating functions.

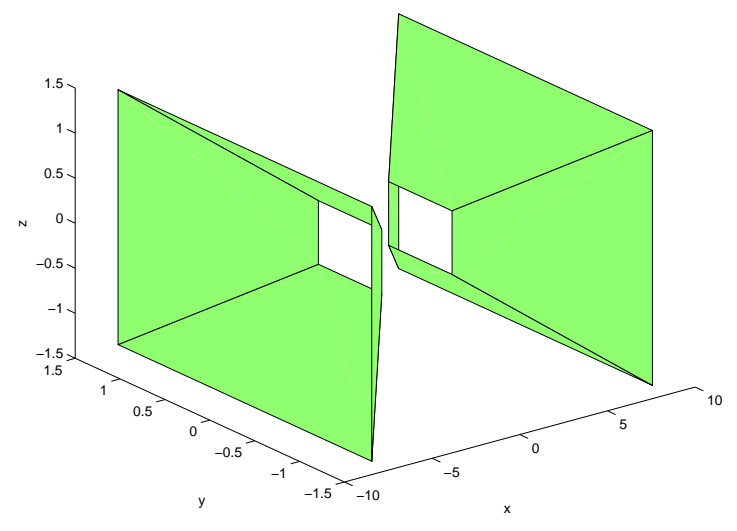

Figure 1. The support of a 3D shearlet $\hat{\varphi}_{a s_{1} s_{2} x}$ in the frequency domain with $a=1 / 4$ and $s_{1}=s_{2}=0$.

To characterize singularities (edge point locations), consider the example of the 3D Heaviside function $H\left(y_{1}, y_{2}, y_{3}\right)=1_{\left\{y_{1}>0\right\}}\left(y_{1}, y_{2}, y_{3}\right)$ where $1_{Y}$ denotes the characteristic function of the set $Y$. It is then known the following are true for $\mathcal{S H}_{\varphi} H\left(a, s_{1}, s_{2}, x\right)=\left\langle H, \varphi_{a s_{1} s_{2} x}\right\rangle$ : 
- If $x=\left(x_{1}, x_{2}, x_{3}\right)$, with $x_{1} \neq 0$, then

$$
\lim _{a \rightarrow 0^{+}} a^{-N} \mathcal{S H}_{\varphi} H\left(a, s_{1}, s_{2}, x\right) \quad \text { for all } \quad N>0 .
$$

- If $s_{1} \neq 0$ or $s_{2} \neq 0$, then

$$
\lim _{a \rightarrow 0^{+}} a^{-N} \mathcal{S H}_{\varphi} H\left(a, s_{1}, s_{2}, x\right) \text { for all } \quad N>0 .
$$

- If $x_{1}=s_{1}=s_{2}=0$, then

$$
\lim _{a \rightarrow 0^{+}} a^{-1} \mathcal{S H}_{\varphi} H\left(a, \bar{s}_{1}, \bar{s}_{2}, x\right) \neq 0 .
$$

This means the continuous shearlet transform of $H$ has rapid asymptotic decay as $a \rightarrow 0$, unless $x$ is on the plane $y_{1}=0$ and $s_{1}, s_{2}$ correspond to the normal direction to the plane. For planes with arbitrary orientation whose normal vector is given as $(\sin \phi \cos \theta, \sin \phi \sin \theta, \cos \phi)$, the continuous shearlet transform will have rapid decay, except for $x$ on the plane and $\left(s_{1}, s_{2}\right)$ satisfying $s_{1}=\tan \theta, s_{2}=\cot \phi \sec \theta$.

In general, let $\Omega$ be a region in $\mathbb{R}^{3}$ with boundary denoted by $\partial \Omega$. We assume the boundary is smooth and has positive Gaussian curvature at every point. If $B=1_{\Omega}$, then we know ${ }^{3}$ :

- If $x \notin \partial \Omega$, then

$$
\lim _{a \rightarrow 0^{+}} a^{-N} \mathcal{S H}_{\varphi} B\left(a, s_{1}, s_{2}, s_{2}, x\right)=0 \quad \text { for all } N>0 .
$$

- If $x \in \partial \Omega$ and $\left(s_{1}, s_{2}\right)$ does not correspond to the normal direction of $\partial \Omega$ at $x$, then

$$
\lim _{a \rightarrow 0^{+}} a^{-N} \mathcal{S} \mathcal{H}_{\varphi} B\left(a, s_{1}, s_{2}, s_{2}, x\right)=0 \quad \text { for all } N>0 .
$$

- If $x \in \partial \Omega$ and $\left(s_{1}, s_{2}\right)=\left(\bar{s}_{1}, \bar{s}_{2}\right)$ corresponds to the normal direction of $\partial \Omega$ at $x$, then

$$
\lim _{a \rightarrow 0^{+}} a^{-1} \mathcal{S H}_{\varphi} B\left(a, \bar{s}_{1}, \bar{s}_{2}, x\right) \neq 0 .
$$

These results establish that edge points can be located by analyzing the asymptotic rate of change of the magnitudes of the 3D shearlet coefficients as a function of scale. This means that the concepts developed in ${ }^{9}$ can be extended and will be valid for 3D shearlets.

\section{IMPLEMENTATION}

Our use of the 3D wavelet and 3D shearlet transforms for edge detection is an extension of the routines developed $\mathrm{in}^{9}$. The horizontal and vertical, and time components of the transform are computed separately and then reassembled in the end. The first critical step for both the wavelet and shearlet processing is to compute the 3D gradient-like components of the image. The continuous scaling is then accomplished by using repeatedly a weighted average filter or mask $A=\left(\begin{array}{lll}1 & 2 & 1 \\ 2 & 4 & 2 \\ 1 & 2 & 1\end{array}\right)$ extended in a similar manner into the third dimension that gives more emphasis to the central pixels. This repeated application of the smoothing filter is used to behave as the continuous dilation of the waveforms. The shearlet directional filtering is accomplished by creating the appropriate frequency formed hyper-trapezoid filters. These directional components are specifically constructed by multiplying the $2 \mathrm{D}$ constructed directional components developed in $^{9}$. A different scale of the image is processed each time the image is convolved with the average filter and represents a different amount of smoothing. This achieves an effect similar to the Canny edge detection process of initially smoothing the image with a Gaussian function at a particular standard deviation. The drawback to the Canny method is choosing the correct standard deviation, as this can be image-specific. Our 3D wavelet and 3D shearlet based methods, on the other hand, can accumulate the gradient information present at multiple scales and thus does not suffer from the same problems as a Canny detector.

It is important to note that the extension of these methods into 3D present some difficult issues. One of these issues is how to appropriately compensate for smoothing that is done in the horizontal, vertical, and time 
directions. Our current method of isolating the edges is accomplished by applying a thresholding step to both the wavelet and shearlet based edge nominations to further refine these nominations and help separate the edge information from noise. Next, a hysteresis thresholding step is applied to both wavelet and shearlet to further refine the edges and help separate edge information from noise. This process includes first setting all values above the high threshold $T_{2}$ to one and setting all values below the low threshold $T_{1}$ to zero. Those values between the high and low threshold are retained only if the pixel is connected to a pixel whose intensity is greater than the high threshold. Finally, a morphological-like process is used to thin the remaining nominated edges by projecting a non-weighted ideal 3D gradient nomination onto the wavelet and shearlet based edge nominations. This method has proven satisfactory, yet we are still in the process of developing better compensation strategies.

\section{EXPERIMENTAL RESULTS}

In this section we compare the results of our $2 \mathrm{D}$ and $3 \mathrm{D}$ wavelet edge detection schemes with the $2 \mathrm{D}$ and $3 \mathrm{D}$ shearlet edge detection schemes. In all experiments, only four scales were processed as this proved adequate. Our experiments use synthetic data to better analyze the performance of the 3D shearlet transform compared to the 3D wavelet transform using known truth data. One of these data sets is a 3D image consisting of a solid spherical harmonic shape located in the center of a cube. The spherical harmonic functions are solutions of Laplace's equation

$$
\nabla^{2} V=0
$$

for spherical polar coordinates. Our experiments used a spherical harmonic of order 2 and degree 7 , shown in Figure 2. Here there are 7 symmetrical structures with 2 lobes on each structure. These functions are

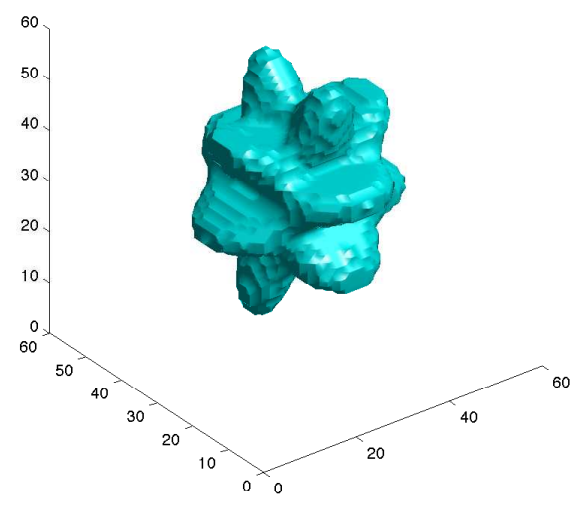

Figure 2. Spherical harmonic truth data

useful because they describe rotation invariant structures for 3D surfaces and present good directionally oriented shapes to test the directional sensitivity of the routines. The first experiment consists of performing the 3D shearlet transform and the 3D wavelet transform based routines on the solid spherical harmonic. This data set was subjected to identically distributed additive white Gaussian noise with a standard deviation $\sigma_{n}=0.2$. To demonstrate the improvements by considering the third dimension, we have also done comparisons with the previously developed 2D routines by applying them separately to a sequence of images creating the dataset. The results are displayed for the wavelet transforms in Figure 3 and for the shearlet transforms in Figure 4 . In this test, the results indicate that the $3 \mathrm{D}$ edge detection routines give a more complete representation of the surface in the presence of noise with fewer artifacts than their 2D counterparts. The contour plots shown only display points with a resulting magnitude of .9 or greater. This presentation has a drawback in not displaying all of the noise present in the result. 

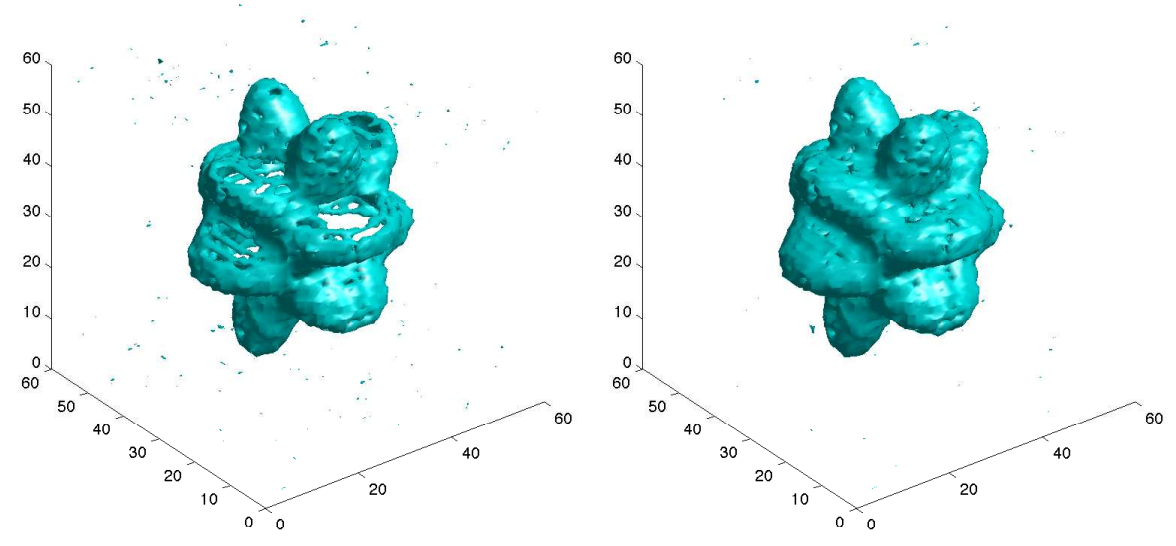

Figure 3. Results for spherical harmonic with noise for 2D (left) and 3D (right) wavelet routines.
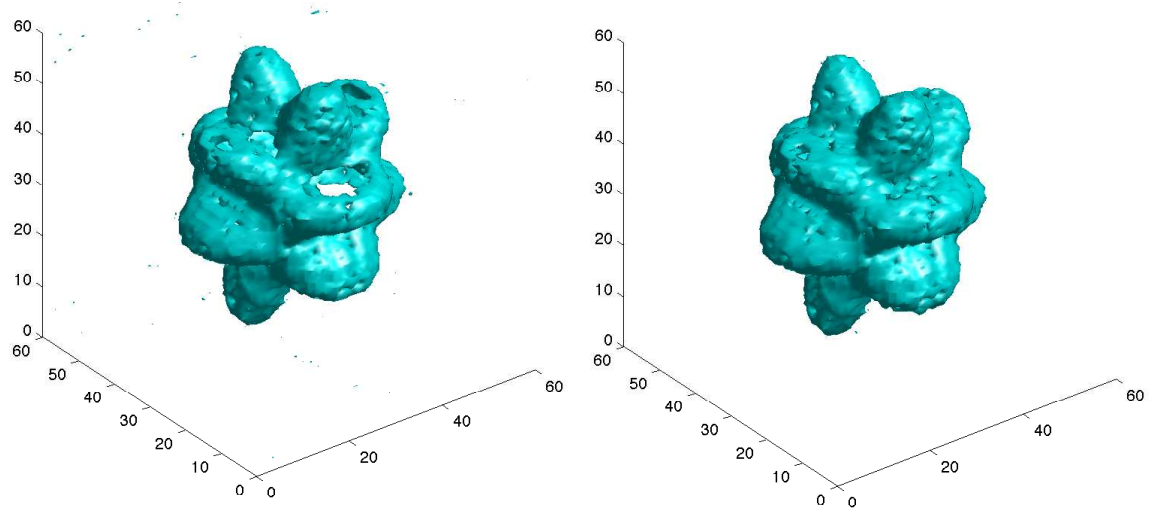

Figure 4. Results for spherical harmonic with noise for $2 \mathrm{D}$ (left) and 3D (right) shearlet routines.

The next experiment involves tracking a solid moving ball of known trajectory through an image sequence. This solid 2D ball spirals around the origin at a given number of frames. A few frames of the image sequence are shown in Figure 5. Both 2D and 3D wavelet transforms are applied to this sequence of images to detect the edges and the results are displayed in Figure 6 . Also both the 2D and 3D shearlet transforms are applied to the same sequence of images to detect the edges and the results are given in Figure 7 .

\section{CONCLUSION}

We have demonstrated the value of applying multi-scale and multi-directional transforms to detect edges in a sequence of images. As expected, extending the traditional 2D wavelet and shearlet transforms to three dimensions has provided more information and improved the detection performance. In particular, the 3D shearlet routine developed so far is especially effective in the presence of noise because it takes edge direction into account. We anticipate further improvements in these routines' performances as we devise better compensation methods for the directional spread we noted earlier. 

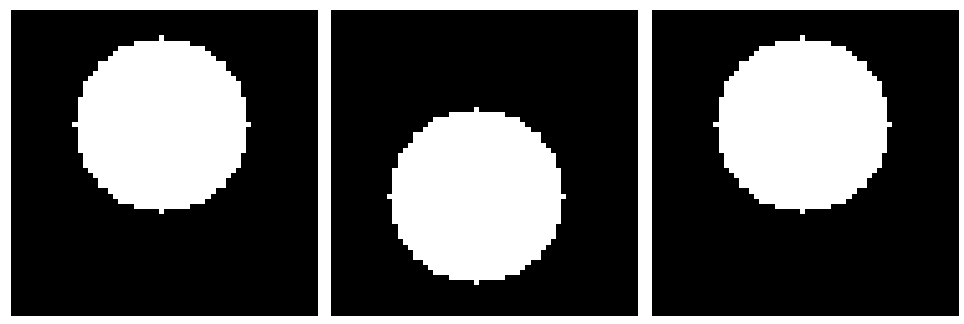

Figure 5. Ball spiraling without noise
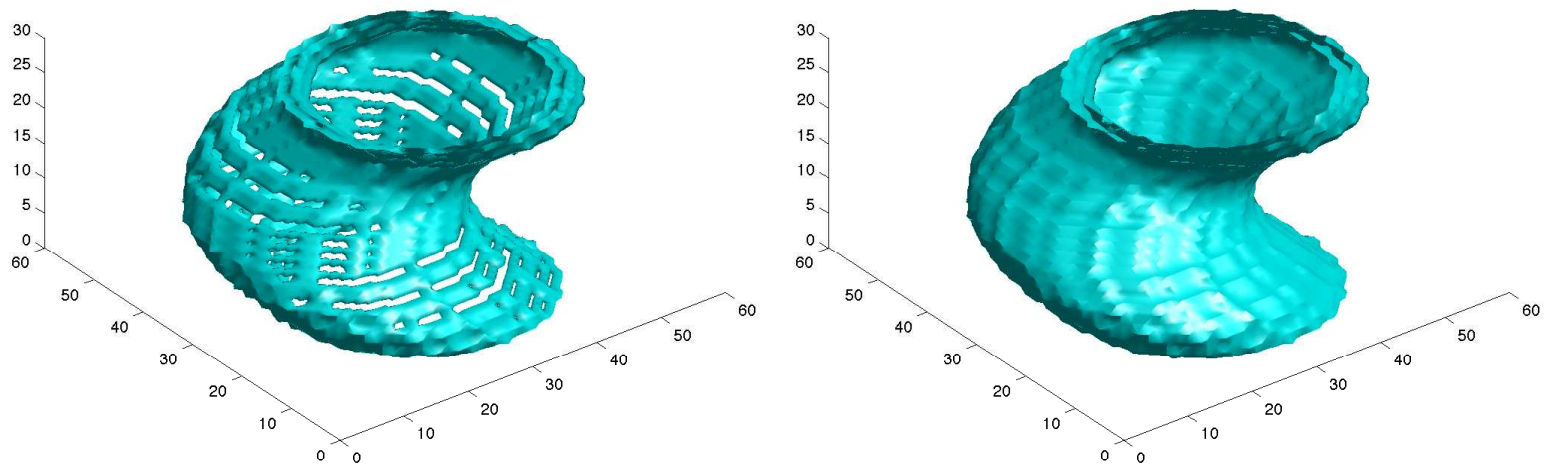

Figure 6. Results of ball spiraling surface detected for $2 \mathrm{D}$ and $3 \mathrm{D}$ wavelet routines without noise added to data.

\section{REFERENCES}

1. G. R. Easley, K. Guo, and D. Labate, "Analysis of Singularities and Edge Detection using the Shearlet Transform", Proceedings of SAMPTA, Marseille 2009.

2. G. R. Easley, D. Labate, and W-Q Lim, "Sparse Directional Image Representations using the Discrete Shearlet Transform", Appl. Comput. Harmon. Anal. 25(1), 25-46, 2008.

3. K. Guo, D. Labate, "Analysis and Detection of Surface Discontinuities using the 3D Continuous Shearlet Transform", Appl. Comput. Harmon. Anal. 231-249, 2010.

4. K. Guo, W. Lim, D. Labate, G. Weiss, E. Wilson, "Wavelets with composite dilations and their MRA properties", Appl. Computat. Harmon. Anal. 20, 231-249, 2006.

5. G. Kutyniok and D. Labate, "Resolution of the Wavefront Set using Continuous Shearlets", Trans. Amer. Math. Soc. 361 2719-2754, 2009.

6. D. Labate, W. Lim, G. Kutyniok and G. Weiss, "Sparse Multidimensional Representation using Shearlets", Wavelets XI (San Diego, CA, 2005), 254-262, SPIE Proc. 5914, SPIE, Bellingham, WA, 2005.

7. O. Monga, R. Deriche, "3D Edge Detection Using Recursive Filtering: Application to Scanner Images", CVGIP: Image Understanding, San Diego, 53(1):76-87, January 1991.

8. S. Yi, D. Labate, G. R. Easley, and H. Krim, "Edge Detection and Processing Using Shearlets", Proceedings IEEE Int. Conference on Image Processing, San Diego, October 12-15, 2008.

9. S. Yi, D. Labate, G. R. Easley, and H. Krim, "A Shearlet Approach to Edge Analysis and Detection", IEEE Trans. Image Proc. 18(5) 929-941, 2009. 

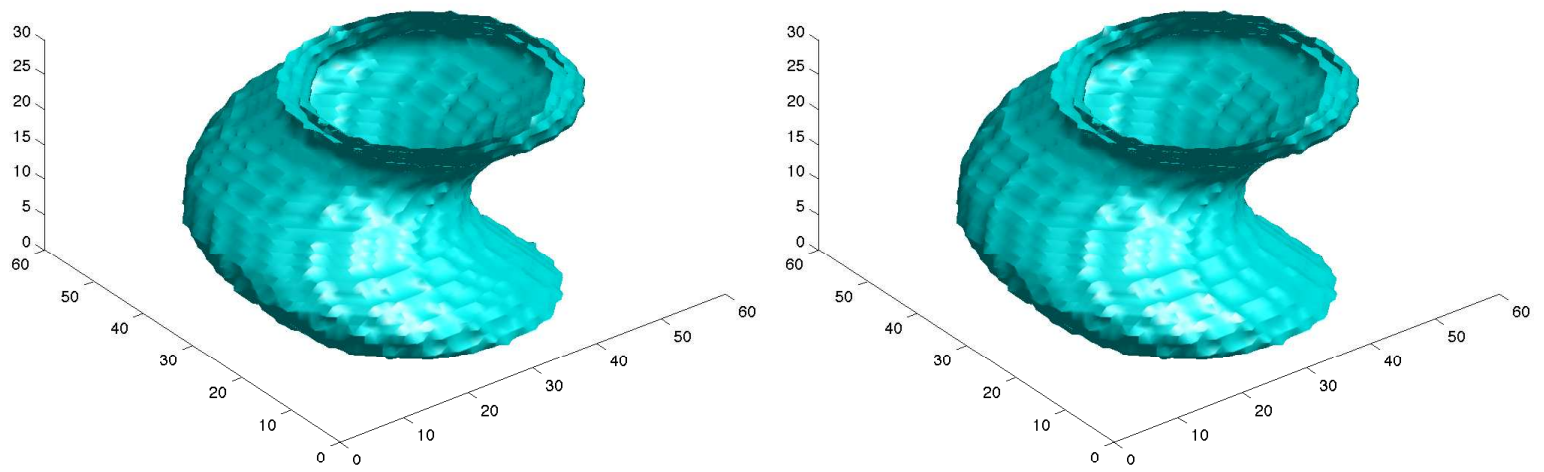

Figure 7. Results of ball spiraling surface detected for $2 \mathrm{D}$ and $3 \mathrm{D}$ shearlet routines without noise added to data.
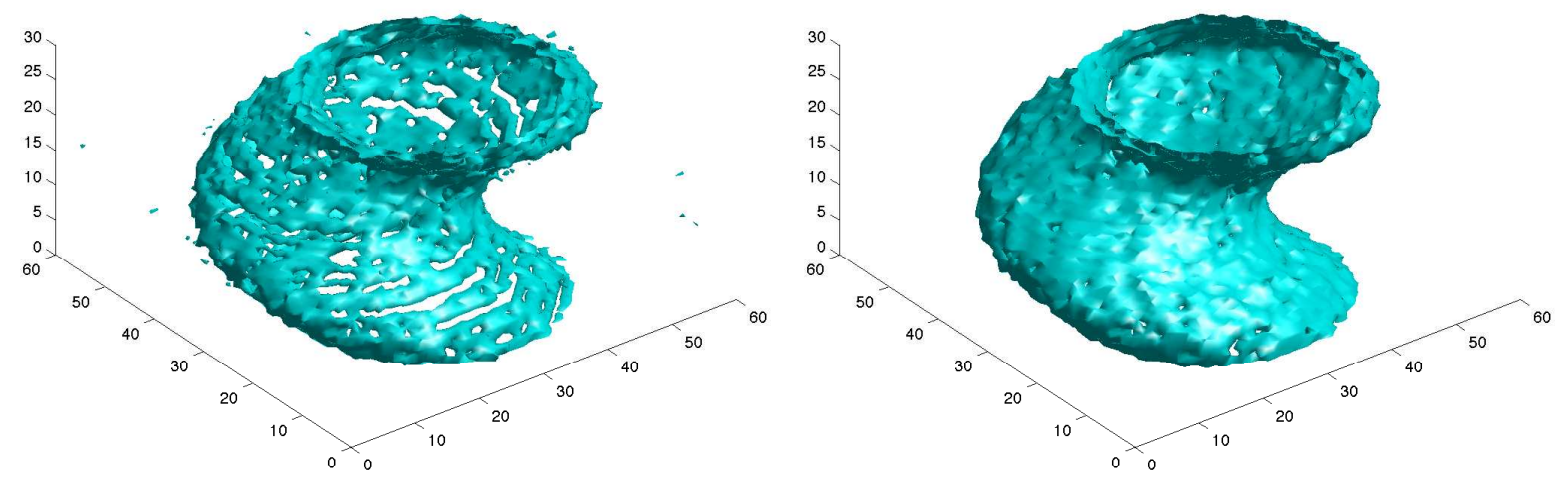

Figure 8. Results of ball spiraling surface detected for 2D and 3D wavelet routines with noise added to data. 

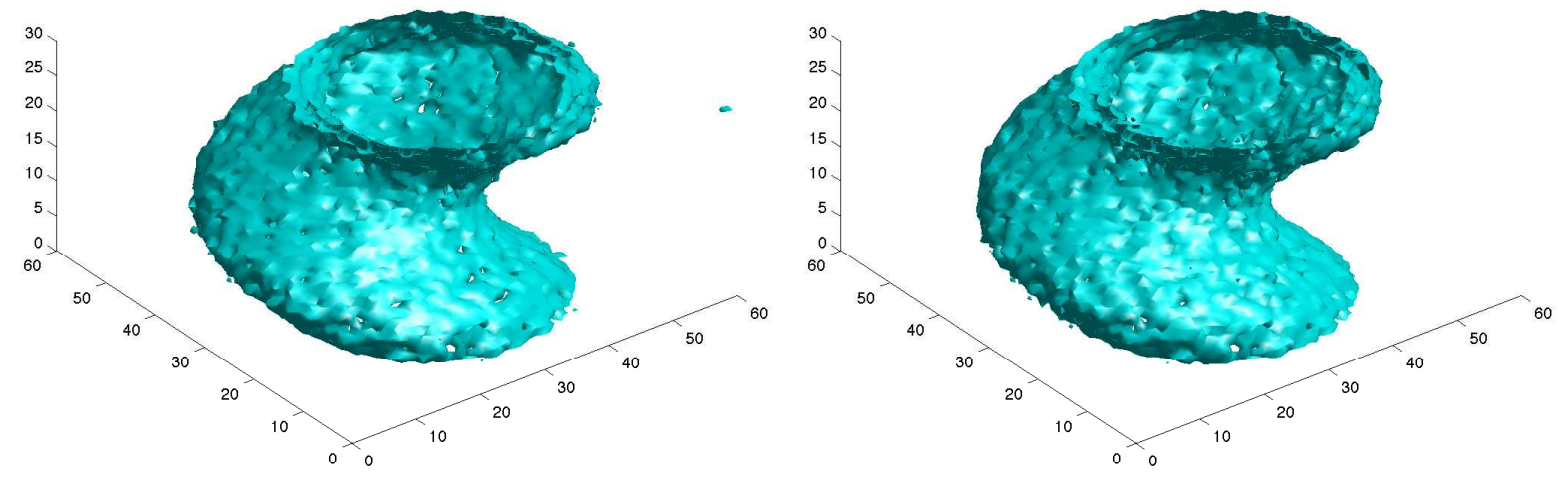

Figure 9. Results of ball spiraling surface detected for $2 \mathrm{D}$ and $3 \mathrm{D}$ shearlet routines with noise added to data. 